\title{
Non-linear long duration blast loading of cylindrical shell structures
}

\author{
*Simon K Clubley \\ BEng(Hons) MBA PhD Eurlng CEng MICE CITP MBCS \\ Lecturer in Civil Engineering, Infrastructure Research Division \\ Faculty of Engineering and the Environment, University of Southampton \\ University Road, Southampton, SO17 1BJ, UK \\ Email: S.K.Clubley@soton.ac.uk \\ Tel. +44 (0) 2380592884
}

Journal submission: September 2013 


\section{ABSTRACT}

This paper investigates the influence of long-duration blast loads on the structural response of aluminium cylindrical shell structures. Full scale coupled non-linear dynamics are examined experimentally at one of the worlds' most powerful air blast testing facilities. Evaluating structural response to blast loads of this magnitude is exceptionally difficult using only computational fluid dynamics; typically not achievable without incurring unmanageable solution domains. Clearing, diffraction and exhaust of a long-duration blast wave across any comparatively small structure imposes constraints leading to the use of approximated drag coefficients, designed primarily to expedite the calculation of net translational forces. In this research, detailed pressure histories measured experimentally on the surface of the cylindrical shell are used to accurately configure a computational analysis dispensing with the requirement to utilise approximated drag forces. When further combined with accurate material test data, fibre optic controlled strain gauge instrumentation and high-speed video photography, a full comparative model was possible. This paper shows that without exact knowledge of long-duration flow-field effects a priori, it is very difficult to reliably determine the mode of structural response and degree of blast resistance. Preliminary modelling predicted a global sway and localised plate buckling; however, subsequent experimental testing showed a crushing failure of the shell before any translational movement occurred. Results in this paper will be of direct interest to both practitioners and researchers considering the dynamic response of cylindrical shell structures subject to high power explosive blasts from sources such as hydrocarbon vapour cloud ignition.

Keywords: cylindrical shell, blast resistance, long-duration, structural response 


\section{Introduction}

Cylindrical shell structures are found in many commercial and industrial settings. Dependent upon their application or use, they may be positioned singularly or in complex arrays of multiple assemblies. The most common occurrence is petrochemical or pharmaceutical. Operationally, the vessels may reside at the end of a long process and act as bulk storage or conversely form part of an in-line chemical engineering activity. Structurally the shells are supported on raised platforms or conversely fully fixed to a base plate. Blast robustness of cylindrical shells is an important consideration to prevent a catastrophic singular or progressive sequence of failure. The source of explosive ignition and shock propagation considered in this paper is unconfined vapour cloud detonation after the flame front. Blast loads of this type are extremely severe and by comparison with conventional explosive, long in time duration approaching 0.5 seconds, as opposed to 10-20 milliseconds [1]. The energy deposition or impulse, in this time frame is considerable; in many cases, the duration of the blast load exceeds the natural mode of the structure and response becomes quasi-static and destabilising [2, 3].

Blast loading to the exterior of a cylindrical shell is an accidental load case imposing statistically severe actions. The 2005 Buncefield vapour cloud explosion in the UK demonstrated the substantial cost of long duration blast damage to both surrounding commercial buildings and industrial equipment [4]. Steel frame and masonry buildings were demolished while shell structures or tanks, used for bulk storage collapsed causing further spillage, fires and secondary explosions. The degree of collapse or plastic deformation depended upon the internal fill depth and any proximity effects to shielding structures. In a number of cases the long duration blast swept away the exterior skin of the shell due to over matching. Structures surrounding the explosion were only designed for a combination of wind and snow load pressures. This is a reasonable and common occurrence given the direction of design codes.

The effects of long duration blast loading on cylindrical shell structures remains an important topic for investigation with little research other than qualitative post failure analysis $[4,5]$ or simplified analytical methods in Baker et al [6] and Kinney [7]. This is in part due to complexities 
in experimentation and analysis encapsulating coupled structural response within a long wavelength flow field $[5,8]$. It was noted by Pritchard et al [5] that damage to structures subject to vapour cloud detonation varies dependent upon whether the range to the explosive centre is very short or at distance. Structures immediately adjacent to the detonation are subject to complex fireball loads unrepresentative of high explosive TNT equivalency; whereas, far field damage demonstrates more similarity due to 'shocking-up' of the blast front. Sources of long duration vapour cloud explosions and their potential mitigation were studied by Nettleton using helium and carbon dioxide curtains [9]. While successful in an experimental construct it remains difficult to deploy around all potentially affected structures, particularly when complex multi-stage detonations can occur as illustrated by Birk et al [10] in the study of two step boiling liquid expanding vapour cloud explosions. This paper specifically considers far field effects on shell structures due to the formation of a near planar shock wave or Mach stem.

Early research examining the dynamic blast response of cylindrical shells and the constituent plates they comprise, focused upon comparatively small explosive sources and elastic behaviour. In many cases, the analytical construct was simplified due to common reasons, (i) resolution of non-linear dynamic response was a formidable task combined with hypothetical load conditions $[11,12]$ and, (ii) experimental constraints dictated that scale models were required i.e. small shock tubes, in which subsequent application to full size structures was difficult [11]. In an attempt to overcome the analytical difficulty, Jiang and Olson [13] modelled the transient response of cylindrical shell structures using a curved finite strip formulation. Focus of the research was directed towards very short blast time durations, leading to impulsive dynamic response only. Transient response of similar cylindrical shells were examined in a theoretical study by Li and Jones [14] and Lellep et al [16] but for short clamped positions with perfectly plastic materials subject to a predetermined cubic yield criterion. Consistent with Jiang and Olson [13] and Pedron and Combescure [15], only small explosive blast loads were considered with the latter evaluating the response of an immersed shell structure.

A number of researchers have considered the localised dynamic response of circular and curved shell plates when subject to blast loading in isolation. The explosive detonation originating 
from both internal and external sources, ranging from military circumstances [17] to unplanned petrochemical process failures [18]. Research remained theoretical in the main with a limited array of miniaturised experiments and hybrid formats [19] in accordance with the Hopkinson scaling law. Neuberger et al [17] reiterated the difficulties involved with conducting full-scale experiments both in terms of cost and technical feasibility.

This paper details the experimental testing of a full scale aluminium cylindrical shell structure subject to long duration blast loading. Conducted in the Air Blast Tunnel facility at Foulness in Essex, UK [20], research examines detailed dynamic structural coupling of the cylinder within the flow field. Using advanced fibre optic instrumentation and high speed Phantom video photography, deformation and collapse of the shell was tracked upon arrival of the shock front and during the sustained dynamic pressure phase. High fidelity numerical modelling used to predict structural response a priori was shown to be inaccurate due to a number of assumptions pertaining to translational drag loads, explored previously by this author [8]. Updated using accurate blast pressure histories from the experiment, computational work demonstrated excellent agreement by comparison with gauge measurements and post-test observations. This research forms part of an on-going test programme examining a range of vessel configurations subject to long duration blast symptomatic of vapour cloud explosive loading.

\section{Experimental Procedure}

The UK Air Blast Tunnel in Essex, UK, designed and constructed originally in 1964 is one of a very small number of elite facilities in the world capable of examining structural response subject to long duration blast loads. The tunnel is approximately $200 \mathrm{~m}$ long with two test regions (i) $4.9 \mathrm{~m}$ diameter section and, (ii) $10.2 \mathrm{~m}$ diameter section. The larger $10.2 \mathrm{~m}$ section allows for experimentation at full-scale in a weather protected environment. Figure 1 shows the tunnel in perspective view from the $10.2 \mathrm{~m}$ exhaust end furthest from the explosive driver source.

The Air Blast Tunnel performance envelope at maximum power comprises a blast shock of $110 \mathrm{kPa}$ with a positive phase duration of 0.2 seconds - preserving safety constraints of the walls [20]. The driver charge producing the near planar blast wave is located in a $1.8 \mathrm{~m}$ diameter section at the start of the tunnel. In essence, the long increasing diameter of the tunnel shapes the blast 
from a conventional high explosive charge profile to that of a long duration pulse. For normal test operation, a near Friedlander type blast wave is generated given the form in Equation 1, [21].

$$
\begin{array}{lll}
p / p_{0} & =\left(1-t / t_{0}\right) e^{-k t / t_{0}} & \text { Eqn }\{1\} \\
\text { Where: } & p & =\text { pressure at time 't' } \\
p_{0} & =\text { peak static overpressure } \\
k & =\text { wave form decay parameter } \\
t_{0} & =\text { arrival time }
\end{array}
$$

Tunnel instrumentation (including pressure gauge, strain gauge, accelerometers and high speed video photography) used to monitor test structures are time sequenced via fibre optics to driver charge detonation at the start of the blast tunnel. Following the experiment in post trial analysis, it is possible to compare the arrival of the shock wave at the structure visually and numerically, with the option to curtail records past the point no longer of interest; thus, saving valuable computer data storage space. Standard environmental gauge monitoring throughout the tunnel length ensures repeatable accuracy of each firing versus the desired test specification.

\subsection{Test Setup}

Full scale cylindrical shell structures are difficult to test from a blast loading perspective due to their curvature and inherent strength; derived from a combined hoop stress and redistribution of loads longitudinally. Despite the immense power of the Air Blast Tunnel, careful pre-trial planning was required to determine a vessel configuration that would respond in the flow field. Importantly, this condition was bounded by a requirement not to record a nil response nor complete collapse and annihilation. A small amount of gauge data would result for the former and large deformation in the latter would near instantaneously clip any findings.

The maximum size cylindrical shell chosen for testing in the planning phase was $4 \mathrm{~m}$ in height to the hemispherical crown lid and $1.4 \mathrm{~m}$ in diameter with an empty fill configuration. The height of vessel was the maximum that could be craned into position while leaving sufficient tunnel roof clearance limiting the effects of potential flow stagnation or choking. A study of increasing fluid fill levels and the potential for shock transmission will be reported in a companion paper. Figure 2 shows a general arrangement of the test structure in the $10.2 \mathrm{~m}$ section. The bottom 
section of the cylinder was painted in a $50 \times 50 \mathrm{~mm}$ square hatched, yellow livery to (i) aid measurement of plate deformation and, (ii) precisely correspond with the mesh density used in numerical modelling. Aluminium alloy grade AA5086- $\mathrm{H} 111$ of thickness $4 \mathrm{~mm}$ was chosen for the shell plates, lid and pre-heated 50mm thick base plate. The use of alloy AA5086-H111 for dynamic impact and response has been investigated for different flat plate thicknesses by Abdulhamid et al [22]. The aluminium metal used for fabrication was independently analysed for mechanical strength properties shown in Table 1.

The bottom shell course was fillet welded to the base plate across the full circumference, simulating full fixity resistant to wind loading effects. Each plate in a shell course was joined by a single vee butt weld of thickness $1.6 \mathrm{~mm}$ at the throat tapering to $22 \mathrm{~mm}$. Plate edges were mechanically cut and file cleaned before the weld was initiated at a maximum interpass temperature of $200^{\circ} \mathrm{C}$ using an Argon gas shield and zirconiated tungsten. Aluminium alloy provided the optimum performance characteristics versus overall size and the safety requirement to mitigate any unsafe choking effects leading to severe tunnel damage. Increased strength of high yield steel combined with the mechanical properties of cylinders was an undesirable choice by comparison with aluminium plate, importantly given the blast tunnel was already configured to fire at maximum power.

The test cylinder was surrounded upstream, alongside and downstream by an array of instrumentation measuring peak static overpressure (PIS1, PIS2 \& PIS3 gauges) and dynamic pressure (PDS' gauges). For simple reference, the positions and offsets are schematically illustrated in Figure 3a (blast arrival from the North side). Dynamic pressure gauges shown in Figure $3 a$ can be noted alongside the vessel in Figure 2 and are shown in more detail in Figure 3b. Height variance of the dynamic pressure stings is recognised by a bottom, middle and top identifier. Structural coupling and response of the shell during blast loading was recorded using Endevco surface mounted, flush pressure gauges. Five were installed on one half of the circumference to monitor the variation in load and response (denoted: PIST1, PIST2, PIST3, PIST4 \& PIST5). Due to the very high cost of each gauge with wiring, monitoring was restricted to 
quadrants. Figure 4 shows the relative compass orientation of each gauge by comparison with the general tunnel instrumentation shown in Figure 3a.

To monitor the local structural behaviour of the cylinder, a series of strain gauges were installed at a minimum $75 \mathrm{~mm}$ height above the base plate. North and south elevations shown in Figure 5, comprised strain rosettes measuring strain in three axes (SNV, SNA \& SNH), where N = north elevation and $\mathrm{V}, \mathrm{A} \& \mathrm{H}=$ vertical, at 45 degrees angle and horizontal orientations respectively. An accelerometer denoted 'ACC', was installed near mid-height on the north side. Figure 6 shows a single vertical strain gauge installed on both east and west elevations (SEV \& SWV) with two high speed photography targets painted on each side. Figure 7 provides a closer view of the attached instrumentation for reference. All of the wiring was kept to a minimum passed through under floor trunking to prevent interference wherever possible.

The blast tunnel was programmed to fire at maximum safe operating power requiring in the larger $10.2 \mathrm{~m}$ section a peak static overpressure of $55 \mathrm{kPa}$ and a dynamic pressure of approximately $8 \mathrm{kPa}$. The intended positive phase duration was 0.2 seconds. Figures $8 \mathrm{a}, 8 \mathrm{~b}$ and $8 \mathrm{c}$ show the recorded pressures in the Air Blast Tunnel versus the required performance envelope; good agreement can be seen for both static and dynamic pressure regimes. Figure 8c provides a comparison of both records for simplicity of reference. The raw pressure history data was recorded during the experiment at a very high sample rate and contained a degree of electrical noise or ringing. These artefacts were filtered for inspection using a standard experimental protocol comprising a fast Fourier transform, low pass smoothing algorithm rationalising a 200 data point sweep.

\section{Numerical Procedure}

Numerical modelling of the cylindrical shell blast response was conducted using LUSAS Finite Element Analysis, Civil and Structural Plus non-linear suite [23]. The solid model shown in Figure 9 matching the physical dimensions of Figures 5 and 6, was used to define the constituent finite element mesh. A thick shell element QTS8 with quadrilateral shape and isoparametric interpolation order was selected to model the aluminium alloy. This element type is suited to threedimensional curved assemblies where transverse shear is present; based on the degeneration of 
a continuum construct. Mesh density of $50 \mathrm{~mm}$ square was assigned matching the yellow hatched livery stuck to the outer shell surface, shown in Figure 2.

Capable of geometric non-linearity, an Updated Lagrangian analysis was selected to model localised buckling and larger scale plate deformations occurring due to continual load destabilisation. Stress and strain calculations used in Updated Lagrangian are based on the Second Piola-Kirchoff stress tensor and the Green-Lagrange strain tensor. Both tensors are capable of large deformation analysis due to invariance under rigid body rotation of the material; suitable for a blast configuration where the structure is continuously updated and evolving [23]. The Green-Lagrange strain measure is shown in Equation 2.

$$
\mathrm{E}=\frac{\mathrm{ds^{2 }}-\mathrm{d5^{2 }}}{2 \mathrm{ds^{2 }}} \quad \text { Eqn }\{2\}
$$

Where: $\quad d S \quad=$ reference configuration for vector length $d X$ undeformed ds = reference configuration for vector length $\mathrm{dx}$ deformed

Aluminium alloy 5086 was defined using a stress potential, total strain hardening plastic material model controlled by a Von Mises yield criterion for a classical continuum formulation. The stress strain characteristics were set to match experimental test data reported in Table 1 with a dynamic strain rate amplification factor of 1.1 for aluminium. For boundary conditions, monolithic full fixity between the aluminium base plate and the bottom shell course was assigned. To replicate passage of the shock wave past the cylinder, flush mounted pressure gauge histories were remapped onto the finite element model using a tapering, quadratic surface field variation controlled in quadrants - as per gauges PIST 1 to 5 , see Figure 4.

A tapering field variation enabled smoothing connectivity between loaded regions compensating for the limitation of flush mounted gauges at the quadrant centre-points, see Figure 10. Staged loading shown in Figure 10 schematically represents the temporal passage of the shock front around the shell circumference; the five diagrams from START to CLEARING - 'E', should be viewed as diagrammatically cumulative and not indicative that earlier pressures are 'switched-off'. Remapped pressure histories from the experiment (corresponding to gauges PIST1 to PIST5, see Figure 4) were parsed directly to the finite element analysis using matching load curves. Local element axis definition was applied as the primary control for the pressure field 
acting normal to the cylindrical shell. The numerical model was solved on a parallel eight CPU workstation with 16GB of RAM and LUSAS fast solver [23]. Typical solution times were approximately 480 dedicated CPU hours.

Solution control for non-linear transient dynamic analysis was set manually in the timedomain for an implicit dynamics integration [23]. Non-convergence termination criteria was applied for a total response time of 0.2 seconds allowing for a full response to a longer duration blast. A small single step time increment was required in the early solution stages, in the order of 2 milliseconds. This was necessary to maintain numerical stability while evaluating a near instantaneous shock front arrival. Implicit dynamics was selected by consideration of the loading speed and degree of impact severity. An implicit solution method requires a stiffness matrix inversion at every time step and as such, is computationally expensive but numerically stable; the Hilber-Hughes-Taylor integration scheme (or $\alpha$-method) is adopted, shown in Equation 3 [23]. Integration using Hilber-Hughes-Taylor permits energy dissipation and second order computational accuracy in time, extending the Newmark approach; also permitting a faster solution scheme with larger time steps. Explicit element settings in LUSAS are more appropriate to numerical models examining very high velocity impacts with lumped masses and consistently small time steps e.g. ammunition round impact; Hilber-Hughes-Taylor with a Jacobian matrix cannot be used in this instance and the Central Difference method is applied.

$$
V_{1}=V_{0}+\left[(1-\gamma) A_{0}+\gamma A_{1}\right] \Delta_{t} \quad \text { Eqn }\{3\}
$$

Where: $\quad V_{0} \quad=$ initial velocity for the initial time step

$A_{0} \quad=$ initial acceleration for the initial time step

$V_{1} A_{1}=$ velocity and acceleration at the first time step

$\Delta_{t} \quad=$ integration time step change

$\gamma=$ Hilber-Hughes-Taylor integration constant

Damping of the numerical model was provided using the Rayleigh Damping Method, or Proportional Method for viscous modal and hysteretic structural effects, shown in Equations 4 \& 5 .

$$
\begin{array}{ll}
C=\alpha M+\beta K & \text { Eqn }\{4\} \\
\xi=0.5\left(\frac{\mu L}{\omega}+\lambda \omega\right) & \text { Eqn }\{5\}
\end{array}
$$




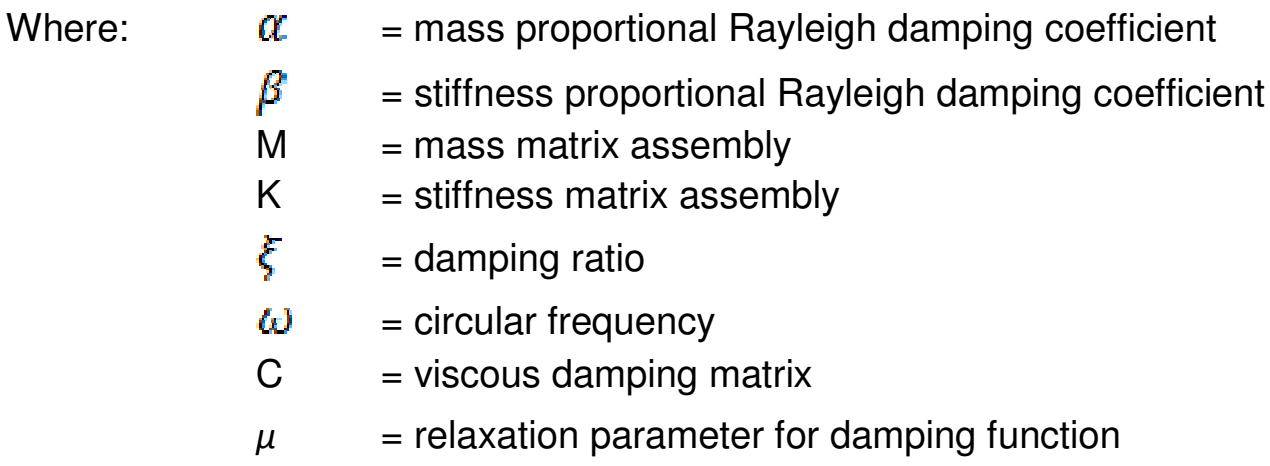

A conservative $1 \%$ damping ' $\lambda$ ', was initially specified for the system based on author experience and the recommendation of the Eurocodes for the design of structures to resist dynamic excitation [24]. A mass Rayleigh damping constant $\alpha$, and stiffness Rayleigh damping constant $\beta$, of 2.92 and $0.31 \times 10^{-3}$ were defined respectively using an eigenvalue modal analysis. Rayleigh damping does not generally affect the first peak response due to blast and so the value selected is initially somewhat arbitrary. Subsequent oscillation and response of the shell structure will be more sensitive to a critical damping determination.

\section{Results and Discussion}

Prior to remapping of pressure histories from the experiment onto the finite element model, a 'best guess' simulation was conducted in the trial planning stage. The numerical model was subject to an initial peak overpressure and a long dynamic pressure phase. The latter applied to the model using a global drag force with a coefficient multiplier in accordance with the work of Clubley [25] and Kinney [7]. It was predicted the vessel would suffer a net translational failure leading to severe local buckling at the base; a subsequent artefact of the load method. Figures $11 \mathrm{a}$ and $11 \mathrm{~b}$ indicate the forecast outcome before the experiment. The crumpled and buckled side of the shell indicating the rear Southern face at PIST5 for reference.

The true temporal distribution of pressure around the cylindrical shell encompassing a coupled structural response was not known a priori and could only be estimated using engineering judgement. Importantly, these complex effects could not be calculated using computational fluid dynamics (CFD) alone, due to the large disparity between the long blast wavelength and the test structure; resulting in an unmanageable cell size structure and solution domain ensuring exhaust of the blast wave [25]. 
Instrumentation in the blast tunnel recorded the reflected and side on pressures experienced by the cylindrical shell as a coupled response. Figure $12 \mathrm{a}$ displays the static overpressure versus the reflected vessel pressures around the circumference. A direct correlation is shown between the free field environment and the near field reflections. Figure $12 \mathrm{a}$ shows that all pressures round the cylinder coalesce to free field values after approximately 0.125 seconds. Figure 12b presents a 'zoomed' scale view of Figure 12a and highlights the staged time of shock arrival around the vessel. Strong, sudden peak shocks are recorded for front facing and side orientated gauges (PIST1 to PIST 3), while comparative gradual rises are shown for rear sectors (PIST4 \& PIST5). Rear facing gauge (PIST5) equalises and maintains a common free field pressure in accordance with PIS2; whereas, PIST4 records lower diffracted pressures due to the angle of obliquity. Side on gauge (PIST3) shows a moderately raised free field pressure compared to PIS2 which subsequently reduces quickly. Figure $12 \mathrm{~b}$ indicates the blast wave required approximately 0.01 seconds to clear the cylindrical shell and stabilise.

Figure 13 displays the horizontal strain rosette results when overlaid against static pressure for reference recorded in $\mathrm{kPa}$, including the aluminium alloy material yield strain. Horizontal hoop strain in the shell remained low during the initial shock arrival phase but increased rapidly on the North side (blast facing) following collapse of the shell front. Yield strain on the North side was exceeded after 50 milliseconds and did not substantially recover thereafter. Strain readings on the protected South side remained low apart from a very rapid initial fluctuation. Figures $14 \mathrm{a}$ and 14b compare the vertical strain readings around the cylinder for all North, South, East and West positions. Figure 14a shows a considerable spike in the PIS2 gauge readings coinciding with a rapid increase in strain for North and South at 0.4 seconds. Figure $14 \mathrm{~b}$ highlights a large compressive longitudinal strain recorded on the South with a high tensile strain on the North side. Twisting of the cylindrical shell produced a high compressive strain exceeding yield in the East side as the vessel leaned reacting to the blast wind. Figures 13 and 14 inclusive, show that load was distributed more effectively in a longitudinal direction with less success transversely around the circumference. 
Progressing from the initial numerical simulations shown in Figures 11a \& 11b, the remapped pressure histories parsed to the finite element model produced results (time sequenced for reference) shown in Figures 15a, 15b and 15c. Figures 15a and 15b highlight an accurate determination of front face collapse leading to large deformation and potential weld fracture in Figure 15c. Comparison of final damage states demonstrate excellent agreement. Using true pressure histories recorded during the experiment removed the requirement to apply a global drag force on the model and speculate: (i) as to an accurate drag coefficient, $C_{D}$ (ii) the projected shell area suitable for application and, (iii) provide adaptive modifications for a changing centre of drag. Figure 16 shows a comparison of strain readings on the North and West sides between experimental data and analytical values. Good agreement is recorded with an informative strain contour plot displayed inset. This accuracy was not possible with the model used to generate Figures 11a and 11b. Three dimensional laser scanning was used following the experiment to record the global and local plate deformation of the cylindrical shell. A Leica laser survey platform recorded cloud point data that could be interrogated for precise comparison. Figure 17a presents a full vessel scan which can be compared with the numerical model shown in Figure 15c. Good agreement was found when a series of cross-section slices were taken through the cylinder depth, shown in Figure 17b. Reasonably, the numerical model was not able to replicate the modest vessel twist slewing from West to East as a product of localised material weakness and weld strength between shell courses.

- Video 1 is supplied for real-time viewing of structural response. The blast wave can be seen to arrive from the left screen edge, clearing to the right side.

\section{Conclusions}

This paper has investigated the coupled non-linear response of a cylindrical shell without liquid fill subject to a high power, long duration blast load. Initial experimental testing has shown that dynamic behaviour is extremely complex and difficult to predict using numerical modelling a priori, without detailed knowledge of the flow field. The mode of structural failure was forecast as a net translational displacement due to the sustained power of the blast and commensurate quasistatic drag force. High speed video photography showed that minor translational movement only 
occurred after the collapse of the front face. Overall, the experimental test structure performed well indicating a substantial degree of damage leading to collapse without becoming disengaged from the base plate. This would cause serious damage to the tunnel rarefaction wave eliminator.

Long duration blast response of the cylindrical shell was successfully modelled using blended finite element analysis techniques. Deformation and failure was accurately captured by comparison with trial results throughout the time history. Remapping of the temporal pressure distribution on the finite element model produced accurate predictions from which an extended parametric study could be further explored. The use of 3D laser scanning in post-trial inspection showed good agreement with the revised predictions of deformed shape. This paper has demonstrated the complexities of long duration blast loading o structures characteristic of large unconfined vapour cloud explosions. Limitations on the calculable domain size for CFD prevents the use of hydrocodes determining the flow field at a useful resolution; a problem compounded when a structure and the flow interact producing a continually updating construct. Only through a blended approach of experimentation and high fidelity finite element analysis can the true effects of unplanned long duration blast events be accurately evaluated.

\section{Acknowledgements}

The author would like to extend gratitude to: the staff at Spurpark Ltd for their help provided during the experimental trials. In addition, the author would like to thank LUSAS FEA for permission to use the software analysis suite. The author acknowledges that all results and data recorded and discussed in this paper are MoD Results obtained at MoD facilities.

\section{References}

[1] SEI, Structural Engineering Institute, 1999. Structural design for physical security, First Edition, ASCE Publishers, p2-10 to 2-17.

[2] Smith P D \& Hetherington J G, 1994. Blast and ballistic loading of structures, First Edition, Butterworth Heinemann Publishers, p52-57.

[3] Cormie D, Mays G \& Smith P D, 2009. Blast effects on buildings, Second Edition, Thomas Telford Publishers, p37-53. 
[4] Atkinson G, 2011. Blast damage to storage tanks and steel clad buildings, Process Safety and Environmental Protection, Vol. 89, pp. 382-390, Elsevier.

[5] Pritchard D K, Roberts A F, 1993. Blast effects from vapour cloud explosions: a decade of progress, Safety Science, Vol. 16, pp. 527-548, Elsevier.

[6] Baker W D, Cox P A, Westine P S, Kulesz J J, Strehlow R A, 1983. Explosion hazards and evaluation, Vol. 5, Elsevier, ISBN 0-444-42094-0.

[7] Kinney G F, 1962. Explosive shocks in air, The Macmillan Company, New York, Library of Congress Catalogue No 62-14792.

[8] Clubley S K, 2013. Steel sections subject to a long duration blast, Journal of Structures and Buildings, Proceedings of the Institution of Civil Engineers, Thomas Telford, in press pending publication.

[9] Nettleton M A, 1976. Alleviation of blast waves from large vapour clouds, Journal of Occupational Accidents, Vol. 1, pp. 3-8, Elsevier.

[10] Birk A M, Davison C, Cunningham M, 2007. Blast overpressures from medium scale BLEVE tests, Journal of Loss Prevention in the Process Industries, Vol. 20, pp. 194-206, Elsevier.

[11] Thompson V K, Ruiz C, 1989. Dynamic elastic and plastic deformation of double-walled cylindrical storage tanks, International Journal of Impact Engineering, Vol. 8, No.4, pp. 341-354, Pergamon.

[12] Aksogan O, Sofiyev A H, 2001. Dynamic buckling of a cylindrical shell with variable thickness subject to a time-dependent external pressure varying as a power function of time, Journal of Sound and Vibration, Vol. 254, No.4, pp. 693-702, Elsevier.

[13] Jiang J, Olson M D, 1991. Nonlinear dynamic analysis of blast loaded cylindrical shell structures, Computers and Structures, Vol. 41, No.1, pp. 41-52, Pergamon.

[14] Li Q M, Jones N, 1995. Blast loading of a short cylindrical shell with transverse shear effects, International Journal of Impact Engineering, Vol. 16, No.2, pp. 331-353, Elsevier.

[15] Pedron C, Combescure A, 1995. Dynamic buckling of stiffened cylindrical shells of revolution under a transient lateral pressure shock wave, Vol. 23, pp. 85-105, Elsevier. 
[16] Lellep J, Torn K, 2004. Plastic response of a circular cylindrical shell to dynamic loadings, International Journal of Impact Engineering, Vol. 30, pp. 555-576, Elsevier.

[17] Neuberger A, Peles S, Rittel D, 2007. Scaling the response of circular plates subjected to large and close range spherical explosions. Part 1: air blast loading, International Journal of Impact Engineering, Vol. 34, pp. 859-873, Elsevier.

[18] Dong Q, Li Q M, Zheng J Y, 2010. Interactive mechanisms between the internal blast loading and the dynamic elastic response of spherical containment vessels, International Journal of Impact Engineering, Vol. 37, pp. 349-358, Elsevier.

[19] Louca L A, Pan Y G, Harding J E, 1998. Response of stiffened and unstiffened plates subjected to blast loading, Engineering Structures, Vol. 20, No. 12, pp. 1079-1086, Elsevier.

[20] Adams J, Rose T A, Garforth R, Evans G, Tate J, 2012. Simulating explosive events in the Air Blast Tunnel, MABS 2012 conference, Bourges France, Crown Copyright.

[21] Friedlander F G, 1939. Note on the diffraction of blast waves by a wall, UK Home Office ARP Dept., RC(A) July, Civil Defence Research Committee Report RC 61.

[22] Abdulhamid H, Kolopp A, Bouvet C, Rivallant S, 2013. Experimental and numerical study of AA5086-H111 aluminium plates subjected to impact, International Journal of Impact Engineering, Vol. 51, Elsevier.

[23] LUSAS Finite Element Analysis, FEA Ltd, version 14.7-5, Civil and Structural Plus, 2013.

[24] BS EN 1991-1-1:2002, Eurocode 1: Actions on structures, Part 1-1General actions, British Standard, BSI, Incorporating corrigenda December 2004 \& March 2009.

[25] Clubley S K, 2013. Steel sections subject to a long-duration blast. Journal of Structures and Buildings, Proceedings of the Institution of Civil Engineers, Thomas Telford, available online ahead of print. 